\title{
Let it be
}

Pierre Bühler

Für Udo

Als uns das Thema dieses Heftes mitgeteilt wurde, kam der Einfall ganz plötzlich, unerwartet: "Let it be«, das Lied der Beatles aus den sechziger Jahren! Und schon verfolgte mich für einen Augenblick die bekannte Melodie. ${ }^{1}$

Doch zunächst leistete ich aktiv Widerstand: "Nein, für diese Nummer wirst Du nichts schreiben; Du bist ja überall bereits im Rückstand! Lass diesen Einfall! - »Let it be« zunächst in einem ganz negativen Sinn: "Lass es doch lieber sein, belaste Dich nicht noch mit dieser zusätzlichen Aufgabe!«

Doch der Einfall wirkte weiter, setzte sich immer mehr gegen meinen Widerstand durch. Eines Tages nahm ich, wie zufällig, die alte Schallplatte aus den sechziger Jahren wieder hervor; später suchte ich nach den Worten ... und fand sie natürlich! Die Beatles krabbelten mir mit ihrem Lied im Geist herum, und ich ahnte immer mehr, dass ich es nicht los werden konnte. Oder besser: dass ich es nur schreibend los werden konnte. "Let it be" nun im Sinne der Ergebung, des Einwilligens: "Lass es sein, lass es geschehen, was da gereift ist."

Schreiben als schreiben lassen?

\section{Warum fand der Einfall Anklang?}

Ein Einfall fällt nicht einfach auf unvorbereiteten Boden. Was hat ihm Resonanz, Anklang, verschafft? Autobiographisch wäre da wohl auf die sechziger Jahre einzugehen: Jugend, Mittelschule, erste grosse Liebe, erste wichtige Lebensverzweigungen, Hoffnung auf wichtige Antworten: "there will be an answer" (und, wie es in einem anderen bekannten Lied hiess, "the answer, my friend, is blowing in the wind «). Als Jugendjahre waren es aber auch "times of trouble«, "hours of darkness". Zeiten, Stunden, in denen die Antwort nicht gleich kam, in denen sich Ungewissheit und Zweifel breitmachten. "And when the night is cloudy, there is still a light that shines on me, shine until tomorrow...«. Und dass es auch für die »broken

\footnotetext{
${ }^{1}$ Die Worte des Liedes finden sich am Ende des Artikels.
} 
hearted people« eine Antwort, eine Chance, gibt, daran wurde heftig geglaubt, darüber wurde eifrig gestritten!

All das schwingt mit beim »Let it be« der Beatles, und dadurch konnte es vielleicht Resonanz in mir finden, in mir einen Schreibprozess auslösen. Aber gibt es noch andere Gründe, die enger mit dem Thema selbst verknüpft sind?

\section{Seinlassen: aktiv oder passiv?}

Das Lied stellt auf neue Weise eine alte Frage. Die Aufforderung "Let it be« erklingt in ihm 26 mal. Zeugt diese fast zwanghafte Wiederholung nicht von einer Art Unmöglichkeit? Kann Seinlassen überhaupt Gegenstand eines Imperativs sein? Muss man das Seinlassen nicht vielmehr einfach geschehen lassen? Das »muss« zeigt jedoch an, dass es sich nicht automatisch einstellt, ohne das Beteiligtsein des Menschen. Dieser muss das Seinlassen in seinem Lebensvollzug wirken lassen. Dass aber »Beteiligtsein« steht, und nicht "Beteiligung", weist darauf hin, dass das Wirkenlassen nicht reine Aktivität ist: Das Seinlassen schafft selbst sein Wirken im Menschen, jedoch so, dass er auch daran beteiligt ist. Deshalb stellt sich die Frage: Aktiv oder passiv? Indikativ oder Imperativ?

Auch im Lied der Beatles ist diese Spannung zu spüren, die Spannung zwischen der Verheissung und der Aufforderung. "There will be an answer, let it be«, heisst es mehrmals. Und wenn »Mother Mary« kommt, spricht sie "Worte der Weisheit" in Gestalt eines Zuspruchs, einer Zusage, aus der das Seinlassen entspringen kann: "Speaking words of wisdom, let it be."

Aus dieser Spannung ist nicht herauszukommen. Indikativ und Imperativ sind nur in diesem Ineinander zu haben, wie sich das biblisch etwa in Philipper 2,12 zeigt: "Mühet euch um euer Heil mit Furcht und Zittern, denn Gott ist es, der in euch sowohl das Wollen als auch das Vollbringen wirkt um seines Wohlgefallens willen." Diese Paulusstelle spielt eine Schlüsselrolle in einem Buch, das demnächst bei Mohr Siebeck herauskommen wird und der Hermeneutik Gadamers gewidmet ist. Der Autor, Philippe Eberhard, unternimmt es, Gadamers Werk von der »Middle Voice«, vom Medium her zu interpretieren (The Middle Voice in Gadamer's Hermeneutics; Hermeneutische Untersuchungen zur Theologie, 45). Das mediale Verbgenus erscheint ihm als eine sprachliche Möglichkeit, die falsche Alternative zwischen aktiv und passiv, zwischen Indikativ und Im- 
perativ, schöpferisch zu überwinden: Das Entscheidende geschieht in einem Prozess, in dem sich etwas passiv erschliesst, an dem der Mensch sich aber zugleich aktiv beteiligt.

Das Seinlassen, wie es bei den Beatles gesungen wird, hat genau diesen medialen Charakter. Es vollzieht sich als ein Prozess zwischen Verheissung und Aufforderung, zwischen einer auffordernden Verheissung und einer verheissungsvollen Aufforderung. Das macht wohl auch seinen weisheitlichen Gehalt aus.

\section{»Words of wisdom»}

Welche Weisheit ist hier gemeint? Wohl nicht ein intellektuelles Wissen oder eine höhere mystische Erkenntnis. Was uns »Mother Mary« hier zuflüstert, ist eher eine Lebensweisheit. Das Seinlassen, zu dem wir eingeladen werden, hat damit zu tun, wie wir mit uns selbst, unserem Leben, unserer Welt umgehen. Wir dürfen all das sein lassen, so wie es ist, mit all seinen Stärken und Schwächen, mit all seinen Schönheiten und Widerlichkeiten. Im Kontrast dazu etwa: Wir müssen nicht aufgehen in einer unendlichen Revolte à la $\mathrm{Ca}$ mus, uns in einer tragischen Auflehnung erschöpfen. Wir müssen uns aber auch nicht mit einer leidenschaftlichen Rechtfertigung des Bestehenden abmühen. „Let it be heisst die weisheitliche Gelassenheit im Umgang mit all dem, was zu uns und zu unserer Welt gehört. Oft geschieht es mir, seit Jahren, dass ich mir »in times of trouble«, "when the night is cloudy«, zuflüstere: »Let it be«, darin befreiende Distanz findend. Aber einem grossen oder kleinen Glück darf dasselbe »Let it be« gelten!

Im klassischen Französisch des 17. Jahrhunderts, bei Leibniz etwa, gab es noch den Begriff der "acquiescence», vom lateinischen Verb "acquiescere«. Er bezeichnet die Einstellung des Zustimmens, des Annehmens, des Gutheissens und deshalb auch des Einwilligens (im Französischen als Verb weiterhin »acquiescer«). Etymologisch ist darin die Wurzel »quies«, »Ruhe«, zu finden: »Acquiescere« meint deshalb ursprünglich "zur Ruhe kommen«, im Schlaf etwa, und metaphorisch auch im Tod. So kann es auf Grabsteinen heissen »Hic acquiescit N. N.", letztes, schlechthinniges Einwilligen zum Ausdruck bringend. Für unsere Thematik ist interessant, dass die "acquiescence« betont, dass ich im Zustimmen, im Gutheissen, Ruhe finde, mich beruhige, mich befriedigt fühle. 
Dass es sich hier auch um eine mediale Lebenserfahrung handelt, kommt am besten beim deutschen Verb "Einwilligen« zum Ausdruck, das von der Wurzel "Wille« her konzipiert ist, aber zugleich, gerade als aktives Verb, das passive Sichergeben dieses Willens meint. Zu einem solchen medialen Einwilligen lädt uns "Mother Mary « ein, und es ist wohl nicht weit von dem entfernt, was man theologisch als Glaube bezeichnet.

\section{Wer ist »Mother Mary«?}

"Mother Mary comes to me, $[\ldots]$ she is standing right in front of me, speaking words of wisdom, let it be." - Wer ist diese "Mutter Maria«, auf die hier verwiesen wird, als Quelle der Weisheit, als Lehrerin des "Let it be«? Die Frage muss wohl offen bleiben, denn das Lied verweigert dem Hörer eine klare Auskunft. Die Phantasie des Theologen gerät jedoch in Bewegung, beeinflusst durch die Nähe adventlicher Stimmung: Könnte es womöglich die zukünftige "Mother Mary« sein, das bescheidene Mädchen, das bei der Ankündigung ihrer künftigen Schwangerschaft dem Engel antwortet: "Es geschehe mir", "fiat mihi« (Lk 1,38)? Auf jeden Fall ist das auch wie ein mediales "Let it be«. Es bringt in einem schlichten Sinn Empfänglichkeit als Einwilligung zum Ausdruck. "Fiat mihi« als ein "acquiescere«, das in der Zustimmung zur Ruhe kommen lässt.

\section{Die Gefahr des Quietismus}

Bei all dem bleibt jedoch ein Unbehagen: Könnte die Gelassenheit der "acquiescence " uns nicht in die Gefahr des Quietismus bringen? Die "quies" könnte sich ethisch tödlich auswirken. Oder um es mit dem »Lassen« auszudrücken: "Let it be« könnte zu einem "Laisserfaire « werden. Im Einwilligen zeigt sich zwar, wie das aktive Wollen sich ergibt. Aus der so gewonnenen Gelassenheit müsste jedoch auch wieder ein aktiver Widerstand hervorgehen.

Mit diesem Problem ringend, betrachte ich erneut das Lied. Dabei zeigt sich plötzlich etwas, das mir bis jetzt entgangen war: Die erste und die dritte Strophe sind in der ersten Person singular geschrieben, das einzelne "Ich" zum Adressaten der "Let it be«Weisheit machend; die zweite Strophe hingegen ist in der dritten Person plural geschrieben, die Aufmerksamkeit auf die "broken hearted people" richtend, für die weiterhin eine Chance bestehe, "though they may be parted«. Damit entsteht plötzlich ein neuer 
Bezug zu »Mother Mary«: Ich hole mir eine andere alte Schallplatte hervor, die ich mir in den sechziger Jahren gekauft habe, meine erste Schallplatte mit klassischer Musik, und spiele sie ab:

$» .$. dispersit superbos ... deposuit potentes ... exaltavit humiles ... esurientes implevit bonis et divites dimisit inanes ...«-Könnten die "words of wisdom« aus Marias Magnificat kommen, als Verheissung für die »broken hearted people«? Wenn dem so ist, wäre im »Let it be« von »Mother Mary« die Kraft eines nüchternen Widerstandes, eines aktiven Einstehens gegeben, das die Gefahr des Quietismus hinter sich lässt, zugleich aber fern von einem unnützen Aktivismus bleibt. Beim Hören des Magnificat stellt sich ein aktives Einstimmen ein, ausgedrückt in einem »Fiat eis!«, »So geschehe es ihnen!«.

\section{»I wake up to the sound of music»}

Von den Beatles war ich also überraschend zu Bach gekommen. Soeben habe ich die "acquiescence« musikalisch als Einstimmen charakterisiert. Einstimmen in das "Seinlassen" wäre damit der musikalisch-mediale Prozess, in dem aus Empfänglichkeit neue, schöpferische Tätigkeit wach werden darf. Arbeit, Einsatz, Mühe, aber in der Zuversicht, dass Gott es ist, "der in euch sowohl das Wollen als das Vollbringen wirkt«.

In diesem Sinne kann ich nun endlich zu meinen Geschäften zurück! 


\section{Die Worte des Liedes}

When I find myself in times of trouble

Mother Mary comes to me,

Speaking words of wisdom, let it be.

And in my hour of darkness

She is standing right in front of me

Speaking words of wisdom, let it be.

Let it be, let it be,

Let it be, let it be,

Whisper words of wisdom, let it be.

And when the broken hearted people

Living in the world agree,

There will be an answer, let it be.

For though they may be parted

There is still a chance that they will see

There will be an answer, let it be.

Let it be, let it be,

Let it be, let it be.

There will be an answer, let it be.

And when the night is cloudy

There is still a light that shines on me,

Shine until tomorrow, let it be.

I wake up to the sound of music -

Mother Mary comes to me,

Speaking words of wisdom, let it be.

Let it be, let it be,

Let it be, let it be.

There will be an answer, let it be.

Let it be, let it be,

Let it be, let it be.

Whisper words of wisdom, let it be. 